\title{
The cardiac phenotype induced by PPAR $\alpha$ overexpression mimics that caused by diabetes mellitus
}

\author{
Brian N. Finck, ${ }^{1}$ John J. Lehman, ${ }^{1}$ Teresa C. Leone, ${ }^{1}$ Michael J. Welch, ${ }^{2,3}$ \\ Michael J. Bennett, ${ }^{4}$ Attila Kovacs, ${ }^{1}$ Xianlin Han, ${ }^{1}$ Richard W. Gross, ${ }^{1,2}$ Ray Kozak, ${ }^{5}$ \\ Gary D. Lopaschuk, ${ }^{5}$ and Daniel P. Kelly ${ }^{1,2,6}$ \\ ${ }^{1}$ Center for Cardiovascular Research, Department of Medicine, \\ ${ }^{2}$ Department of Molecular Biology and Pharmacology, and \\ ${ }^{3}$ Department of Radiology, Washington University School of Medicine, St. Louis, Missouri, USA \\ ${ }^{4}$ Department of Pathology, University of Texas Southwestern Medical Center and Children's Medical Center of Dallas, \\ Dallas, Texas, USA \\ ${ }^{5}$ Departments of Pediatrics and Pharmacology, University of Alberta, Edmonton, Canada \\ ${ }^{6}$ Department of Pediatrics, Washington University School of Medicine, St. Louis, Missouri, USA
}

Address correspondence to: Daniel P. Kelly, Center for Cardiovascular Research, Washington University School of Medicine, 660 S. Euclid Avenue, Campus Box 8086, St. Louis, Missouri 63110, USA.

Phone: (314) 362-8908; Fax: (314) 362-0186; E-mail: dkelly@imgate.wustl.edu.

Received for publication August 27, 2001, and accepted in revised form November 12, 2001.

\begin{abstract}
Recent evidence has defined an important role for PPAR $\alpha$ in the transcriptional control of cardiac energy metabolism. To investigate the role of PPAR $\alpha$ in the genesis of the metabolic and functional derangements of diabetic cardiomyopathy, mice with cardiac-restricted overexpression of PPAR $\alpha$ (MHC-PPAR) were produced and characterized. The expression of PPAR $\alpha$ target genes involved in cardiac fatty acid uptake and oxidation pathways was increased in MHC-PPAR mice. Surprisingly, the expression of genes involved in glucose transport and utilization was reciprocally repressed in MHC-PPAR hearts. Consistent with the gene expression profile, myocardial fatty acid oxidation rates were increased and glucose uptake and oxidation decreased in MHCPPAR mice, a metabolic phenotype strikingly similar to that of the diabetic heart. MHC-PPAR hearts exhibited signatures of diabetic cardiomyopathy including ventricular hypertrophy, activation of gene markers of pathologic hypertrophic growth, and transgene expression-dependent alteration in systolic ventricular dysfunction. These results demonstrate that (a) PPAR $\alpha$ is a critical regulator of myocardial fatty acid uptake and utilization, (b) activation of cardiac PPAR $\alpha$ regulatory pathways results in a reciprocal repression of glucose uptake and utilization pathways, and (c) derangements in myocardial energy metabolism typical of the diabetic heart can become maladaptive, leading to cardiomyopathy.
\end{abstract}

J. Clin. Invest. 109:121-130 (2002). DOI:10.1172/JCI200214080.

\section{Introduction}

Diabetes mellitus is associated with increased cardiac morbidity and mortality (1). Cardiomyopathy occurs commonly in diabetics independent of known risk factors such as coronary artery disease or hypertension (2). Although little is known about the pathogenesis of diabetic cardiomyopathy, evidence is emerging that it may be related to derangements in myocardial energy metabolism $(3,4)$. Under normal conditions, myocardial energy substrate preference varies in a dynamic manner to fulfill the tremendous energy needs of the postnatal mammalian heart. Whereas the fetal heart relies primarily on glucose and lactate, the capacity for mitochondrial fatty acid oxidation (FAO) increases markedly after birth, providing the adult heart the option of using glucose or fatty acids to meet energy demands depending on dietary and physiologic conditions (5). In diabetes, the capacity for cardiac energy substrate switches becomes constrained due to the importance of insulin in the control of myocardial glucose uptake and utilization. In the uncontrolled diabetic state, the heart relies almost exclusively on FAO for its ATP requirements $(3,4)$. This alteration in myocardial energy substrate utilization could have detrimental consequences such as increased myocardial oxygen consumption due to excessively high mitochondrial oxidative flux. In addition, diabetic hearts import fatty acids at a high rate (6-8), resulting in myocardial lipid accumulation which predisposes the myocytes to death and contractile dysfunction $(9,10)$.

Cardiac fatty acid utilization pathways are controlled, in part, at the gene regulatory level. Recent studies have demonstrated an important role for the nuclear receptor peroxisome proliferator-activated receptor $\alpha$ $(\operatorname{PPAR} \alpha)$ in the transcriptional control of genes involved in cardiac fatty acid uptake and oxidation (reviewed in ref. 11). PPAR $\alpha$, a ligand-activated transcription factor, binds cognate DNA regulatory elements as a heterodimeric partner with the 9-cis retinoic acid receptor RXR. Long-chain fatty acids and a variety of related compounds serve as PPAR $\alpha$ ligands. Genes encoding FAO enzymes and proteins involved in cellular fatty acid import have been identified as PPAR $\alpha$ targets (11). Basal expression of peroxisomal and mito- 
chondrial FAO enzymes is reduced in hearts of PPAR $\alpha$ null mice (12-14). PPAR $\alpha$ has also been shown to activate cardiac and hepatic FAO enzyme gene expression during fasting when FAO rates increase $(15,16)$.

Recently, the PPAR $\alpha$ gene regulatory pathway has been implicated in the hepatic metabolic response to diabetes mellitus. The expression of PPAR $\alpha$ is elevated in the livers of mice rendered diabetic by streptozotocin (STZ) (17) or leptin receptor deficiency $(d b / d b)(18)$. Furthermore, the hepatic induction of PPAR $\alpha$ target genes in response to STZ-induced insulin deficiency was found to be absent in PPAR $\alpha$-null mice (19). These results suggest that one of the mechanisms whereby cellular FAO rates are increased in the diabetic state occurs via activation of the PPAR $\alpha$ gene regulatory pathway. In this study, mice with cardiac-specific overexpression of PPAR $\alpha$ were produced so that the metabolic consequences of increased PPAR $\alpha$ activity and its effects on cardiac metabolism and function could be evaluated in the absence of systemic alterations known to occur in the diabetic state.

\section{Methods}

Animal studies and production of MHC-PPAR transgenic mice. Diabetes was induced by a single intraperitoneal injection of STZ (Sigma Chemical Co., St. Louis, Missouri, USA; $180 \mathrm{mg} / \mathrm{kg}$ body weight). Diabetes was confirmed by tail blood glucose monitoring (B-GLUCOSE Analyzer; Hemacue AB, Angelholm, Sweden). Mice with blood glucose of greater than $250 \mathrm{mg} / \mathrm{dl}$ were considered diabetic. Male C57/BLKS/J-Lepr ${ }^{d b}$ $(d b / d b)$ and heterozygote $(d b /+)$ littermate mice were obtained from The Jackson Laboratory (Bar Harbor, Maine, USA). At 10 weeks of age, mice were sacrificed for tissue collection.

A cDNA construct containing a 1.5-kb, $\mathrm{NH}_{2}$-terminal FLAG epitope-tagged PPAR $\alpha$ cDNA was cloned downstream of the cardiac $\alpha$ myosin heavy chain (MHC) promoter (clone 26; gift of J. Robbins, The Children's Hospital Research Foundation, Cincinnati, Ohio, USA). Transgenic mice were produced by microinjection of the MHC-PPAR $\alpha$ construct into fertilized one-cell $\mathrm{C} 57 \mathrm{BL} / 6 \times \mathrm{CBA} / \mathrm{J} \mathrm{F}_{1}$ embryos. Functional and metabolic endpoints were analyzed in pairs of MHC-PPAR and littermate nontransgenic (NTG) mice ranging in age from 8 to 16 weeks (22-30 g body weight).

Wy-14,643 (BIOMOL Research Laboratories Inc., Plymouth Meeting, Pennsylvania, USA) was incorporated into standard rodent chow ( $0.1 \%$ weight/weight; Bio-Serv Inc., Frenchtown, New Jersey, USA). Mice were given ad libitum access to matched control chow (no. F3028, Bio-Serv Inc.) or Wy-14,643-containing chow and killed for sample collection 1 week later.

For the fasting studies, male and female littermate mice were separated into individual cages at the beginning of each experiment. Mice were fasted for 24 hours. Control mice were allowed ad libitum access to standard laboratory chow (Diet 5053; Purina Mills Inc., St. Louis, Missouri, USA).
All animal experiments were approved by the Animal Studies Committee of Washington University School of Medicine.

Micro-positron emission tomography studies. For micro-positron emission tomography (microPET) analyses of cardiac ${ }^{11} \mathrm{C}$-palmitate and ${ }^{18} \mathrm{~F}$-fluorodeoxyglucose $\left({ }^{18} \mathrm{~F}-\mathrm{FDG}\right)$ uptake, mice were fasted for 6 hours and then anesthetized using isoflurane. A sterile microcatheter was placed within the external jugular vein. The first imaging sequence was initiated, and after a 3 -second delay, a bolus injection of $0.3 \mathrm{mCi}$ of ${ }^{11} \mathrm{C}$-palmitate was administered via the jugular catheter. MHC-PPAR and NTG littermate mice were imaged side by side with the microPET-R4 camera (Concorde Microsystems Inc., Knoxville, Tennessee, USA) for 40 minutes. After data acquisition, there was a 30-minute "wash-out" followed by a second scan after a bolus injection of $0.5 \mathrm{mCi}$ of ${ }^{18} \mathrm{~F}-\mathrm{FDG}$.

Mouse isolated working heart preparation. Mouse working heart perfusions were carried out as described by Belke et al. (20). Working hearts were perfused with Krebs-Henseleit solution containing $5 \mathrm{mM}$ glucose, $100 \mu \mathrm{U} / \mathrm{ml}$ insulin, and $0.4 \mathrm{mM}$ palmitate. Myocardial fatty acid and glucose oxidation rates were determined by the quantitative collection of ${ }^{3} \mathrm{H}_{2} \mathrm{O}$ or ${ }^{14} \mathrm{CO}_{2}$ produced by hearts perfused with buffer containing $\left[9,10-{ }^{3} \mathrm{H}\right]$ palmitate or $\left[\mathrm{U}-{ }^{14} \mathrm{C}\right]$ glucose $(20)$.

Echocardiographic studies. Transthoracic M-mode and two-dimensional echocardiography was performed on conscious mice using an Acuson Sequoia 256 Echocardiography system (Acuson Corp., Mountain View, California, USA) as described (10).

Histologic analyses. After harvest, a midventricular slice of myocardium was snap-frozen in a cryomold for sectioning. To detect neutral lipid, the sections were stained with oil red $\mathrm{O}$ and counterstained with hematoxylin.

$R N A$ and protein blot analyses. The protocols for total RNA isolation and Northern blotting have been described (13). Cardiac ventricle was used as the source for RNA isolation unless noted otherwise. Northern blot analyses were performed using the radiolabeled cDNA probes described in the text. Band intensities were quantified by phosphorimaging analysis and normalized to the expression of GAPDH.

For Western blotting studies to detect PPAR $\alpha$, cardiac protein extracts were prepared as described (21) using an antibody directed against murine PPAR $\alpha$ (gift of J.W. Woods and J.P. Berger, Merck Research Laboratories, Rahway, New Jersey, USA). Total cell membrane protein extracts were prepared as described (10) for use in immunoblot studies with anti-acyl-CoA oxidase (anti-ACO), anti-GLUT1, anti-GLUT4, and anti-actin (Sigma Chemical Co.).

Determination of carnitine palmitoyltransferase I enzymatic activity. Carnitine palmitoyltransferase I (CPT I) activity was determined in isolated cardiac mitochondria from MHC-PPAR and NTG mice by measuring the formation of palmitoyl- $\left[{ }^{14} \mathrm{C}\right]$-carnitine from palmitoylCoA and $\left[{ }^{14} \mathrm{C}\right]$-carnitine as described (22). 
Analysis of myocardial triacylglycerol levels. The quantitative analysis of myocardial triacylglycerol (TAG) species has been described (23). In brief, lipids were extracted from mouse heart using a modified Bligh and Dyer technique in the presence of internal standards for electrospray ionization mass spectroscopic (ESIMS) analysis. ESIMS analyses of TAG were performed using a Finnigan TSQ-7000 spectrometer (Finnigan Corp., San Jose, California, USA) equipped with an electrospray ion source as described previously (23).

Serum chemistries. Serum nonesterified fatty acid (NEFA), TAG, glucose, and insulin levels were determined by enzymatic, colorimetric assays by the Core Laboratory of the General Clinical Research Center at Washington University School of Medicine.

Statistical analysis. Statistical comparisons were made using ANOVA coupled to Scheffe's test. All data are presented as means \pm SEM, with a statistically significant difference defined as a $P$ value less than 0.05 .

\section{Results}

The PPAR $\alpha$ gene regulatory pathway is activated in the diabetic mouse heart. We sought to determine whether the increased rates of myocardial FAO known to occur in the diabetic heart are associated with activation of the PPAR $\alpha$ gene regulatory pathway. Northern blot analyses were performed using total RNA isolated from ventricular tissue of mice rendered diabetic for 6 weeks by administration of STZ. The expression of the genes encoding PPAR $\alpha$ and its cardiac-enriched coactivator PPAR $\gamma$ coactivator 1 (PGC-1) was increased in hearts of STZ-treated mice compared with vehicle-injected littermates (Figure 1a). In addition, the expression of PPAR $\alpha$ target genes encoding mitochondrial (muscletype CPT; M-CPT I) and peroxisomal (ACO) FAO enzymes was upregulated two- to threefold in the hearts of mice treated with STZ.

The expression of PPAR $\alpha$ target genes was also activated in hearts of diabetic $d b / d b$ mice, which are obese and severely insulin-resistant due to a leptin receptor defect. The expression of PPAR $\alpha$-responsive genes (ACO and M-CPT I) was elevated in the hearts of $d b / d b$ mice compared with $d b /+$ littermate controls (Figure $1 b)$. In contrast to the insulin-deficient mice, PPAR $\alpha$ gene expression was not altered in $d b / d b$ mouse heart, but levels of PGC-1 mRNA were significantly increased compared with those in controls (Figure 1b). Taken together, these data indicate that the cardiac PPAR $\alpha$ regulatory pathway and downstream genes involved in cellular FAO are activated in insulin-deficient and insulin-resistant forms of diabetes mellitus.

Production of transgenic mice with cardiac-restricted overexpression of PPAR $\alpha$. We hypothesized that chronic activation of the cardiac PPAR $\alpha$ regulatory pathway would lead to cardiac energy metabolic derangements and functional abnormalities similar to the diabetic heart. To establish a mouse model with constitutively increased activity of cardiac PPAR $\alpha$, transgenic mice were generated in which FLAG epitope-tagged PPAR $\alpha$ was overexpressed in postnatal cardiac ventricle via the cardiac $\alpha$ myosin heavy chain gene promoter (MHCPPAR mice). Four independent lines of MHC-PPAR transgenic mice were established (404-3, 402-2, 404-11, and 404-4) exhibiting a wide range of cardiac-restricted transgene expression (Figure 2a). PPAR $\alpha$ protein levels were approximately 135-fold (404-3), 80-fold (402-2), 40-fold (404-11), and 15-fold (404-4) more abundant in the cardiac ventricles of transgenic animals compared with NTG littermates. Transgenic mice from each of the lines bred normally and litter sizes were similar to those of NTG mice. The 6-month mortality of the transgenic mice was not different from that of NTG littermates.

Increased expression of PPAR $\alpha$ target genes involved in cardiac FAO in MHC-PPAR mice. The expression of PPAR $\alpha$ target genes involved in FAO was characterized in the hearts of MHC-PPAR mice. Levels of mRNA encoding ACO were significantly higher (approximately
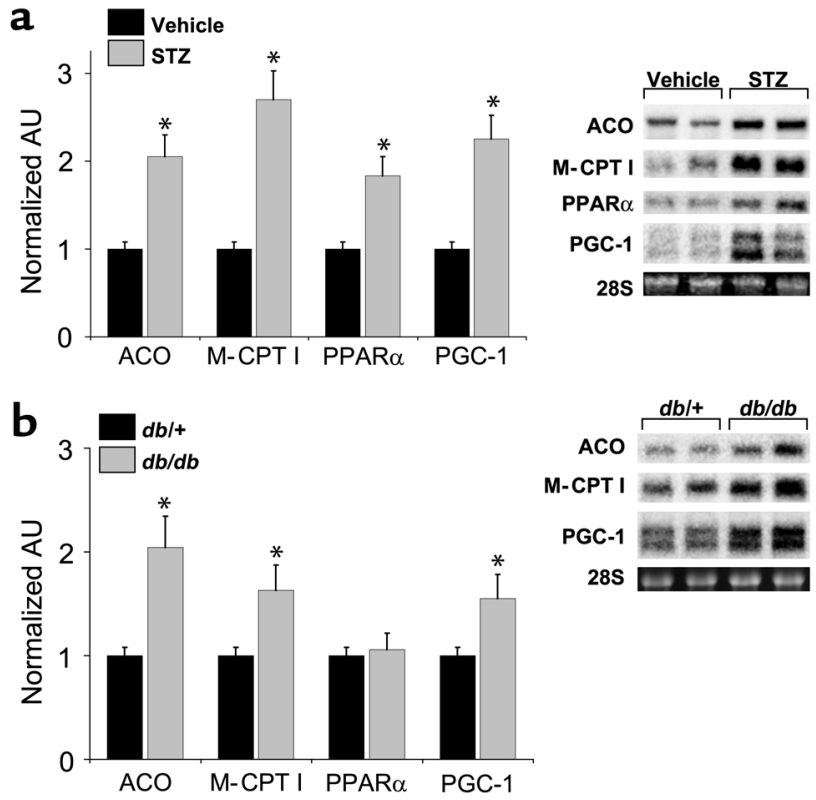

\section{Figure 1}

Increased expression of PPAR $\alpha$ target genes in diabetic mouse myocardium. (a) Bars represent mean ( \pm SEM) mRNA levels as determined by phosphorimager analysis of Northern blots performed with RNA isolated from mouse ventricle, shown as arbitrary units $(A U)$ corrected for GAPDH signal intensity and normalized to the value of vehicle-injected controls $(=1.0)$. ${ }^{*} P<0.05$ versus vehicle-injected mice ( $n=4$ for each group). A representative autoradiograph of the Northern blot studies is shown at the right. Each lane contained $15 \mu \mathrm{g}$ total RNA isolated from mice 6 weeks after an injection of saline (vehicle) or a single dose of STZ $(180 \mathrm{mg} / \mathrm{kg})$. The blot was sequentially hybridized with the radiolabeled cDNA probes shown on the left. (b) Increased expression of PPAR $\alpha$ target genes in $d b / d b$ diabetic mice. Bars represent mRNA levels shown as arbitrary units $(A U)$ corrected for GAPDH signal intensity and normalized to the value of $d b /+$ littermate controls $(=1.0)$. ${ }^{*} P<0.05$ versus $d b /+$ littermate controls; $n=5$ for each group. A representative autoradiograph is shown at the right. Each lane contained $15 \mu \mathrm{g}$ of ventricular total RNA isolated from 10-week-old heterozygote control $(d b /+)$ or littermate diabetic $(d b / d b)$ mice. 
a

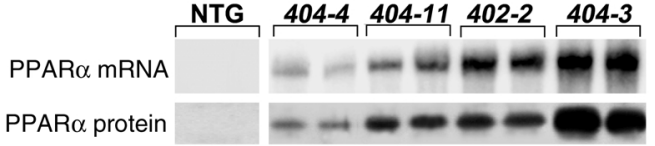

FLAG-PPAR

H BAT SM K L

b

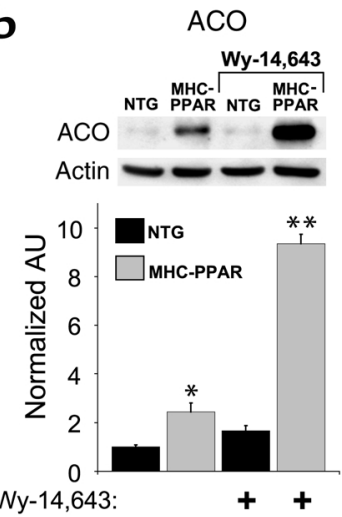

M-CPT I

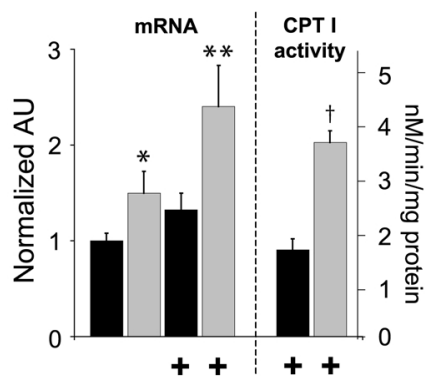

Figure 2

Increased expression of PPAR $\alpha$ target genes in hearts of transgenic mouse lines (MHC-PPAR mice) that express PPAR $\alpha$ in a cardiacrestricted manner. (a) Representative autoradiographs of Northern (top) and Western (middle) blot studies performed with heart samples from 6-week-old mice from four independent MHC-PPAR lines. At the exposure shown, endogenous PPAR $\alpha$ could not be detected in NTG samples. FLAG-PPAR $\alpha$ protein was detected in the transgenic mice using an antibody directed against either PPAR $\alpha$ (shown here) or FLAG (data not shown). Cardiac-specific expression of FLAGPPAR $\alpha$ mRNA was confirmed by Northern blot analysis performed with multiple tissues (bottom panel represents the 402-2 line). $\mathrm{H}$, heart; BAT, brown adipose tissue; SM, skeletal muscle; K, kidney; L, liver. (b) The expression of PPAR $\alpha$ target genes is increased and inducible by PPAR $\alpha$ activator in the hearts of MHC-PPAR mice. The graphs represent the results of Northern blot analyses performed with the probes indicated using RNA isolated from ventricle of male and female NTG or MHC-PPAR littermate mice fed control chow or a 7-day course of chow containing Wy-14,643 (0.1\% wt/wt). Bars represent mean mRNA levels (arbitrary units, AU) quantified as described in Methods ( $n \geq 7$ mice for each group). The inset shows representative autoradiographs of Western blot analyses. ${ }^{*} P<0.05$ versus NTG littermate mice. ${ }^{*} P<0.05$ versus MHC-PPAR mice fed control chow and NTG mice. Far right: Bars represent mean malonylCoA-inhibited CPT enzyme activity ( $n=3$ for each group). ${ }^{\dagger} P<0.05$ versus NTG littermate mice.

twofold) in myocardium of the highest (404-3) and lowest (404-4) transgene-expressing lines of MHCPPAR mice than in NTG littermate controls (Figure 2b and Table 1). M-CPT I mRNA levels were also higher in the hearts of MHC-PPAR (404-3) mice than in the hearts of NTG controls (Figure $2 \mathrm{~b}$ and Table 1). To investigate the possibility that PPAR $\alpha$ ligand was limiting for full activation of its target genes in MHCPPAR mice, ACO and M-CPT I gene expression was characterized in MHC-PPAR and NTG littermate mice fed the synthetic PPAR $\alpha$ activator Wy-14,643. In NTG mice, administration of $\mathrm{Wy}-14,643(0.1 \%$ by weight in chow) for 7 days resulted in the marked induction of

ACO mRNA expression in liver (data not shown), but not in heart (Figure 2b). In contrast, Wy-14,643 caused a robust (over ninefold) induction of ACO gene expression in hearts of MHC-PPAR (404-3) mice (Figure 2b and Table 1). ACO protein levels were also increased in MHC-PPAR (404-3) hearts (Figure 2b). Interestingly, the induction of ACO protein levels was more robust than that observed for the corresponding mRNA levels in MHC-PPAR mice (Figure $2 \mathrm{~b}$ and data not shown). Wy-14,643-mediated induction of cardiac ACO gene expression was greater in the highest transgene expressing line (404-3) than in the lowest expressing line (404-4), suggesting that ligand is limiting for full activation of this PPAR $\alpha$ target gene in MHCPPAR mice. However, M-CPT I mRNA levels were induced to a similar extent (about twofold) by PPAR $\alpha$ activator in both 404-3 and 404-4 lines, suggesting that additional factors are limiting for the PPAR $\alpha$-mediated transcriptional activation of this gene. CPT I enzymatic activity was also increased in MHC-PPAR heart compared with controls (Figure $2 \mathrm{~b}$ ). The levels of malonyl-CoA, an endogenous inhibitor of CPT I, were not significantly different in the hearts of NTG and MHC-PPAR mice (data not shown). Given that ACO and M-CPT I catalyze rate-limiting steps in peroxisomal and mitochondrial $\beta$-oxidation, respectively, these results indicate that the capacity for cellular FAO is increased in the hearts of MHC-PPAR mice.

Recent studies have shown that the mitochondrial uncoupling protein UCP3 is a PPAR $\alpha$ target (24) and that its expression is increased in the hearts of STZ-induced diabetic rats (25). Accordingly, the cardiac expression of the UCP2 and UCP3 genes was evaluated in the MHCPPAR mice. The levels of UCP2 mRNA (nearly twofold) and, to a greater extent, UCP3 mRNA (approximately fivefold) were significantly greater in MHC-PPAR heart than in that of NTG controls (Table 1).

Activation of the cardiac fatty acid uptake pathway in MHCPPAR mice. The expression of PPAR $\alpha$ target genes encoding proteins involved in cellular fatty acid import (fatty acid transport protein 1 [FATP1] and CD36) and thioesterification (fatty acyl-CoA synthetase 1; FACS1) was also evaluated in MHC-PPAR mice. At baseline, FATP1, CD36, and FACS1 mRNA levels were not significantly different in MHC-PPAR heart (404-3 and 4044) compared with controls. However, Wy-14,643 administration significantly induced the cardiac expression of each in MHC-PPAR mice, but not in NTG controls (Table 1). The inducible overexpression of FATP1, CD36, and FACS1 in MHC-PPAR mice suggested that, under physiologic circumstances in which PPAR $\alpha$ ligand levels rise, myocardial fatty acid uptake would increase, possibly beyond the capacity for oxidation. Indeed, a histologic signature of the diabetic heart is myocyte lipid accumulation due to increased myocyte fatty acid import. Fasting is known to increase circulating NEFA levels, leading to activation of PPAR $\alpha$ in heart and liver $(15,16)$. Therefore, the histologic appearance of myocardium from MHC-PPAR and 
Table 1

PPAR $\alpha$ target gene expression in MHC-PPAR mice

\begin{tabular}{lcccc}
\cline { 2 - 2 } 404-3 & \multicolumn{2}{c}{ Control chow } & \multicolumn{2}{c}{ Wy-14,643 chow $(0.1 \%)$} \\
Gene & NTG & MHC-PPAR & NTG & MHC-PPAR \\
M-CPTI & $1.0 \pm 0.25$ & $1.5 \pm 0.11^{\mathrm{A}}$ & $1.4 \pm 0.13$ & $2.3 \pm 0.20^{\mathrm{A}}$ \\
ACO & $1.0 \pm 0.20$ & $2.4 \pm 0.13^{\mathrm{A}}$ & $1.4 \pm 0.32$ & $9.4 \pm 0.07^{\mathrm{A}}$ \\
FATP1 & $1.0 \pm 0.30$ & $1.0 \pm 0.09$ & $1.6 \pm 0.42$ & $1.9 \pm 0.24^{\mathrm{A}}$ \\
FACS1 & $1.0 \pm 0.20$ & $1.3 \pm 0.18$ & $1.5 \pm 0.15$ & $3.2 \pm 0.40^{\mathrm{A}}$ \\
CD36 & $1.0 \pm 0.32$ & $1.1 \pm 0.22$ & $0.89 \pm 0.45$ & $2.4 \pm 0.30^{\mathrm{A}}$ \\
UCP2 & $1.0 \pm 0.25$ & $1.9 \pm 0.26^{\mathrm{A}}$ & $2.0 \pm 0.40$ & $2.6 \pm 0.45$ \\
UCP3 & $1.0 \pm 0.33$ & $5.0 \pm 0.10^{\mathrm{A}}$ & $4.6 \pm 0.11$ & $6.1 \pm 0.06^{\mathrm{A}}$ \\
404-4 & & & & \\
M-CPT & $1.0 \pm 0.10$ & $1.3 \pm 0.15$ & $0.9 \pm 0.28$ & $2.0 \pm 0.30^{\mathrm{A}}$ \\
ACO & $1.0 \pm 0.25$ & $2.0 \pm 0.40$ & $1.6 \pm 0.21$ & $4.2 \pm 0.82^{\mathrm{A}}$ \\
FACS1 & $1.0 \pm 0.30$ & $1.3 \pm 0.30$ & $2.1 \pm 0.11$ & $3.1 \pm 0.31^{\mathrm{A}}$ \\
CD36 & $1.0 \pm 0.21$ & $1.5 \pm 0.24$ & $0.9 \pm 0.23$ & $2.7 \pm 0.13^{\mathrm{A}}$
\end{tabular}

Values represent mean $( \pm$ SEM) arbitrary units normalized to that of the NTG mice $(=1.0)$ without $\mathrm{Wy}-14,643$ treatment based on phosphorimager analysis of Northern blot studies ( $n \geq 7$ for each group). ${ }^{\text {ASignificant difference }(P<0.05)}$ compared with NTG littermate within the corresponding treatment group.

NTG mice was evaluated following a short-term fast. In the fasted state, oil red O staining revealed substantial neutral lipid droplet accumulation in cardiac myocytes from MHC-PPAR mice, whereas NTG littermates remained mainly oil red O-negative (Figure 3a). Cardiac sections from the nonfasted MHC-PPAR and NTG groups were negative for neutral lipid (data not shown). To quantify and characterize the lipid accumulation in MHC-PPAR hearts, myocardial lipid was extracted from fed and fasted transgenic and NTG littermate mice for ESIMS analysis. The myocardial lipid content in MHC-PPAR mice was not significantly different from that of NTG controls under nonfasted conditions (Figure $3 \mathrm{~b}$ ). However, as predicted by the oil red $\mathrm{O}$ staining pattern, myocardial TAG levels were markedly higher in fasted MHC-PPAR mice than in controls. Levels of long-chain fatty acid-containing TAG (16:0, 18:1, 18:2) were dramatically increased in fasted MHCPPAR mice compared with NTG mice (Figure 3b). Although the quantity of myocardial TAG was much

\footnotetext{
Figure 3

Myocardial lipid accumulation in fasted MHC-PPAR mice. (a) Photomicrographs depicting the histologic appearance of ventricular tissue from NTG and MHC-PPAR mice following a 24-hour fast at low (upper panels) and high (lower panels) magnification. Frozen tissue sections were stained with oil red $\mathrm{O}$. Red droplets indicate neutral lipid staining. (b) Lipid profile of mouse ventricle samples prepared from MHC-PPAR or NTG mice given ad libitum access to food or fasted for 24 hours. Lipid species were separated and analyzed using ESIMS (see Methods). Mass/charge $(\mathrm{m} / \mathrm{z}$ ) ratios of 814,840 , and 864 denote TAGs containing fatty acyl groups containing chain lengths of 16:0/16:0/16:0, 16:0/16:0/18:1, and 16:0/18:1/18:2, as shown at the top. (c) Representative autoradiographs of Northern blot analyses performed with total RNA isolated from cardiac ventricle of mice fed ad libitum or after a 24-hour fast using cDNA probes for diacylglyceride acyltransferase (DGAT), glycerol-3-phosphate acyltransferase (GPAT), and adipophilin. Ethidium bromidestained 28S rRNA is shown as a control for loading.
}

greater in MHC-PPAR mice, the profile of TAG species was not different between NTG and MHC-PPAR mice (Figure 3b). Serum NEFA levels were similar in NTG and MHC-PPAR mice in both the fasted and the fed states (fed, $0.80 \pm 0.04$ vs. $0.86 \pm 0.02 \mu \mathrm{M}$, respectively; fasted, $1.34 \pm 0.18$ vs. $1.44 \pm 0.15 \mu \mathrm{M}$, respectively), indicating that the supply of circulating NEFA was equivalent in both genotypes.

The observed increase in neutral lipid content in the fasted MHC-PPAR myocardium suggested that pathways involved in TAG synthesis were induced. The myocardial expression of genes encoding glycerol-3phosphate acyltransferase (GPAT) and diacylglycerol acyltransferase (DGAT), two key enzymes involved in the esterification of fatty acids to TAG, was significantly increased by PPAR $\alpha$ overexpression at base line and further with fasting (Figure $3 \mathrm{c}$ ). The cardiac expression of adipophilin, a putative fatty acid-binding protein that associates with lipid droplets, was also increased in MHC-PPAR mice (Figure 3c). Surprisingly, the cardiac expression of the genes encoding the fatty acid import proteins FATP1, FACS1, and CD36 was not significantly greater in the fasted MHC-PPAR mice than in NTG mice (data not shown).

Reduced expression of cellular glucose import proteins and utilization enzymes in the hearts of MHC-PPAR mice. A metabolic signature of the diabetic heart is reduced

a

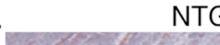
NTG

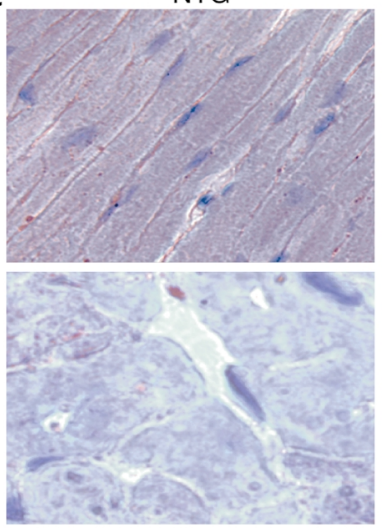

b
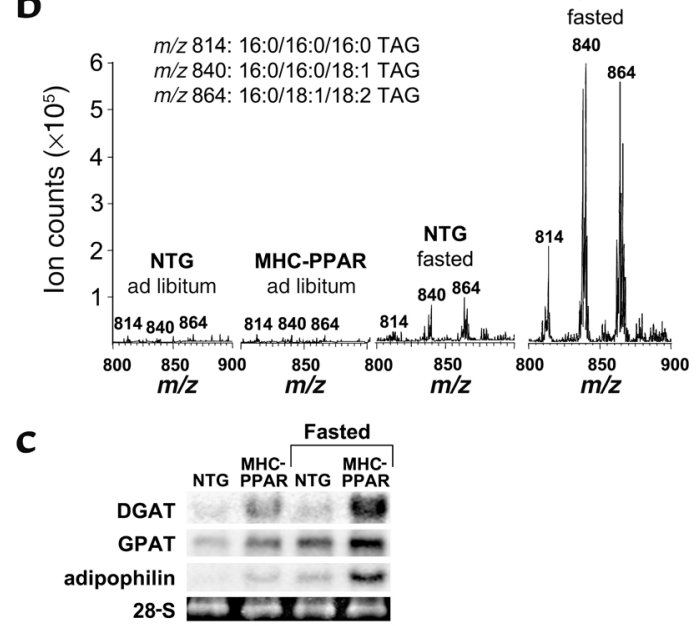

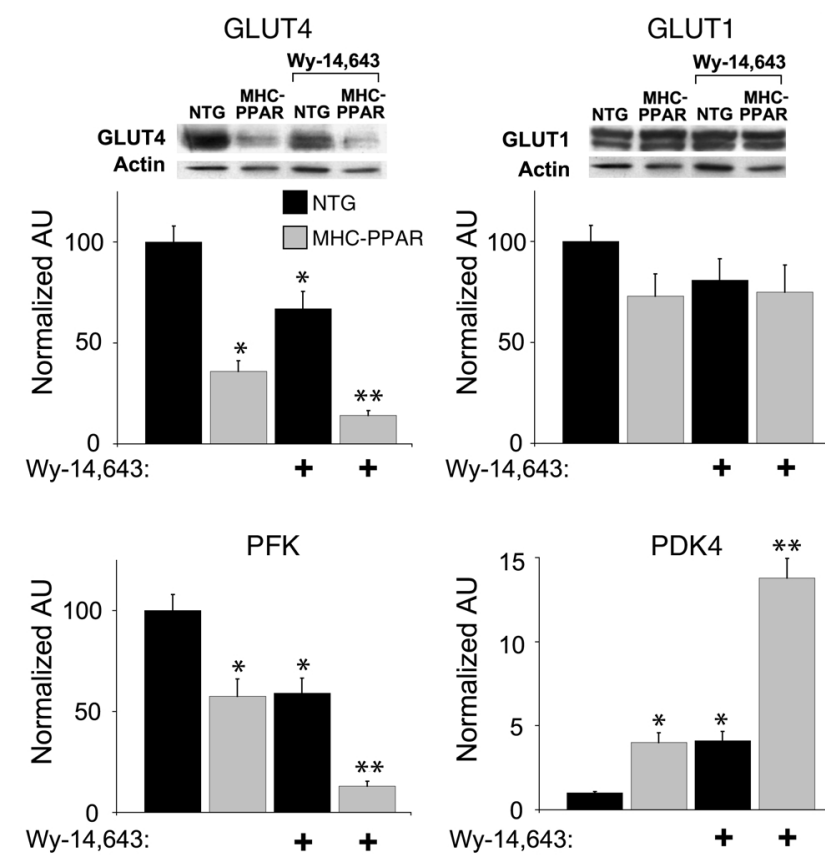

\section{Figure 4}

Altered expression of genes involved in glucose utilization pathways in MHC-PPAR heart. The graphs represent the results of Northern blot analyses performed with total RNA isolated from the cardiac ventricles of MHC-PPAR or littermate NTG mouse hearts. The blots were sequentially hybridized with radiolabeled cDNA probes for GLUT4, GLUT1, PFK, and pyruvate dehydrogenase kinase 4 (PDK4). Bars represent mean mRNA levels as determined by phosphorimager analysis ( $n \geq 7$ for each group) corrected to the GAPDH signal and normalized to that of the NTG mice treated with control chow $(=100$ or 1.0). Groups receiving a 7-day course of $W y-14,643$ are denoted at the bottom of each graph. Top: Representative autoradiographs of Western blot analyses using anti-GLUT4 or anti-GLUT1 antibodies with protein extracts containing total cell membrane-enriched fractions of cardiac ventricle from MHC-PPAR mice and NTG littermates. ${ }^{*} P<0.05$ versus NTG littermate mice fed control chow. ${ }^{*} P<0.05$ versus MHC-PPAR mice fed control chow and NTG mice. Bars represent the mean of at least seven individual animals.

glucose uptake and utilization $(3,4,26)$. The myocardial expression of genes encoding glucose transporters (GLUT1 and GLUT4) and the glycolytic enzyme phosphofructokinase (PFK) was evaluated in MHC-PPAR mice. Levels of GLUT4 mRNA were markedly lower (over 50\%) in hearts of 404-3 MHC-PPAR mice than in those of controls (Figure 4). Treatment with Wy-14,643 resulted in a further downregulation of GLUT4 gene expression in MHC-PPAR mice and in NTG mice (Figure 4). Immunoblot analysis also revealed a striking reduction $(70-75 \%)$ in GLUT4 protein levels in total cell membrane-enriched protein extracts prepared from hearts of MHC-PPAR mice compared with controls (Figure 4). In contrast, GLUT1 mRNA and protein levels were not affected by PPAR $\alpha$ overexpression at base line or following Wy-14,643 treatment (Figure 4). PFK gene expression was also reduced in MHC-PPAR hearts at base line and by Wy-14,643 treatment (Figure 4). These data indicate that the expression of genes involved in insulin-responsive glucose transport and glycolysis is downregulated in the MHC-PPAR heart.

The expression of pyruvate dehydrogenase kinase 4 (PDK4), a negative regulator of glucose oxidation via its inhibitory effects on the pyruvate dehydrogenase complex, was recently shown to be increased in diabetic heart (27). Compared with untreated NTG controls, cardiac PDK4 mRNA levels were fourfold higher in MHC-PPAR mice fed control chow and 14-fold higher after PPAR $\alpha$ activator treatment (Figure 4). Wy-14,643 treatment also led to a fourfold activation of PDK4 expression in NTG mice. Cardiac PDK4 protein levels were also higher in MHC-PPAR mice (data not shown). Taken together, these data indicate that multiple steps in the cellular glucose utilization pathway are inhibited at the gene regulatory level in the MHC-PPAR heart.

Altered myocardial fatty acid and glucose uptake and utilization rates in MHC-PPAR mice. The results of the gene expression studies predicted that fatty acid uptake is increased and glucose uptake reciprocally decreased in the hearts of MHC-PPAR mice compared with NTG controls. To investigate myocardial fatty acid and glucose import rates in vivo in MHC-PPAR mice, microPET studies were performed. The myocardial uptake of ${ }^{11} \mathrm{C}$-palmitate was increased in MHC-PPAR (404-3 line) mice compared with NTG littermate controls (representative image, Figure 5a). Conversely, ${ }^{18} \mathrm{~F}-\mathrm{FDG}$ uptake was substantially lower in hearts of MHC-PPAR mice than in those of NTG littermates. Time-activity curves demonstrated that accumulation of ${ }^{11} \mathrm{C}$-palmitate into the MHC-PPAR heart was significantly increased and ${ }^{18} \mathrm{~F}-\mathrm{FDG}$ accretion decreased for 20 minutes following bolus injection of the labeled substrates (Figure $5 \mathrm{a})$. Serum NEFA ( $0.80 \pm 0.04$ vs. $0.86 \pm 0.02 \mu \mathrm{M})$, glucose $(197 \pm 14.6$ vs. $189 \pm 12.5$ $\mathrm{mg} / \mathrm{dl})$, and insulin $(0.82 \pm 0.25 \mathrm{vs} .0 .67 \pm 0.21 \mathrm{ng} / \mathrm{ml})$ levels were similar in MHC-PPAR and NTG littermate mice, respectively.

To assess myocardial substrate utilization in MHCPPAR mice, the oxidation of $\left[9,10-{ }^{3} \mathrm{H}\right]$ palmitate and $\left[\mathrm{U}-{ }^{14} \mathrm{C}\right]$ glucose was measured in working hearts isolated from MHC-PPAR or NTG littermate mice. We found that the mean rate of $\left[{ }^{3} \mathrm{H}\right]$ palmitate oxidation was $64 \%$ higher in hearts isolated from MHC-PPAR (402-2 line) mice than in NTG hearts $(294 \pm 44$ vs. $179 \pm 9$ $\mathrm{nmol} / \mathrm{min} / \mathrm{g}$ dry weight; $P<0.05$; Figure $5 \mathrm{~b}$ ). Strikingly, mean glucose oxidation rates were diminished by $63 \%$ in MHC-PPAR hearts compared with controls $(302 \pm 150$ vs. $824 \pm 72 \mathrm{nmol} / \mathrm{min} / \mathrm{g}$ dry weight; $P<0.05$; Figure $5 \mathrm{~b}$ ). These data, which are consistent with the results of the gene expression and PET studies, indicate that hearts of MHC-PPAR mice depend on fatty acid for the generation of energy to a significantly greater extent than do hearts of NTG mice, a substrate utilization profile similar to that of the diabetic heart.

Cardiac hypertrophy and ventricular dysfunction in MHCPPAR mice. The initial stages of diabetic cardiomyopathy are characterized by ventricular hypertrophy and diastolic dysfunction (28). In severe cases, cardiac 
abnormalities can progress to systolic ventricular dysfunction and overt congestive heart failure. As an initial step to determine whether the metabolic derangements resulting from PPAR $\alpha$ overexpression lead to cardiac dysfunction similar to that of diabetic cardiomyopathy, hearts from age- and sex-matched mice from all four MHC-PPAR lines were examined for signs of ventricular hypertrophy compared with NTG littermates. MHC-PPAR mice from each of the four lines exhibited an increased biventricular weight/body weight ratio in a pattern that was generally dependent upon the level of transgene expression (Figure 6a). Histologic examination of left ventricular (LV) tissue sections stained with hematoxylin and eosin or trichrome revealed no obvious abnormalities in myocardial cytoarchitecture, except for myocyte hypertrophy (3-month-old MHC-PPAR mice; data not shown). The expression of hypertrophic growth marker genes braintype natriuretic peptide (BNP), atrial natriuretic factor (ANF), and skeletal $\alpha$-actin was induced in ventricle of 6-week-old 404-3 MHC-PPAR mice (Figure 6b) and in less highly expressing lines (data not shown). In contrast, the ratio of cardiac $\alpha$ myosin heavy chain to $\beta$ myosin heavy chain mRNA levels was not significantly different between the two groups (data not shown). As has been reported in the diabetic heart (25, $29)$, the expression of the genes encoding sarcoplasmic/endoplasmic reticulum $\mathrm{Ca}^{2+}$ ATPase 2a (SERCA2a) and its regulatory protein phospholamban (PLB) was reduced in MHC-PPAR hearts (Figure 6b). Echocardiography was performed on animals from all four MHC-PPAR lines. As shown in Figure $6 c$ and Table 2, posterior wall thickness was increased in the 404-3 and 402-2 lines of MHC-PPAR mice. LV chamber dilatation, as determined by measurement of systolic (LVIDs) and diastolic (LVIDd) internal diameters, was increased in the most highly expressing line of MHCPPAR mice (404-3)compared with littermate controls. Fractional shortening was significantly reduced in the three most highly expressing lines compared with NTG littermates (Table 2; Figure 6c). These data indicate that MHC-PPAR mice develop cardiac hypertrophy and ventricular dysfunction in a transgene expression level-dependent manner.

\section{Discussion}

The energy metabolic alterations associated with diabetic cardiomyopathy include excessive rates of myocardial fatty acid uptake and oxidation coupled with reduced glucose utilization. In an attempt to model the metabolic derangements of the diabetic heart, we have generated mice with cardiac-specific overexpression of the nuclear receptor PPAR $\alpha$. This strategy was employed so that the impact of altered myocardial energy metabolism on cardiac function could be assessed in the absence of the systemic diabetic state. We found that the MHC-PPAR mice exhibit a cardiac metabolic phenotype that is strikingly similar to that of the diabetic heart. Several lines of evidence support this conclusion.
First, as in the diabetic heart, the expression of genes involved in the myocardial fatty acid utilization pathway is activated in MHC-PPAR hearts. Second, a reciprocal downregulation of the myocardial glucose transport, glycolytic, and glucose oxidation pathways occurs in the transgenic mice. Third, fatty acid uptake and oxidation rates are increased whereas glucose uptake and oxidation is abnormally suppressed in the hearts of MHC-PPAR mice. Lastly, the transgenic mice exhibit evidence of altered cardiac myocyte lipid balance as has been described for the diabetic heart.

a
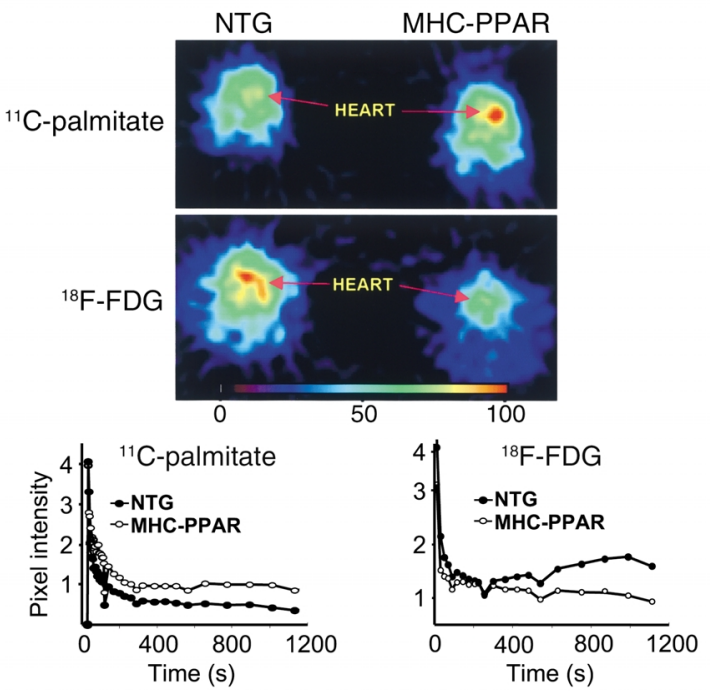

b
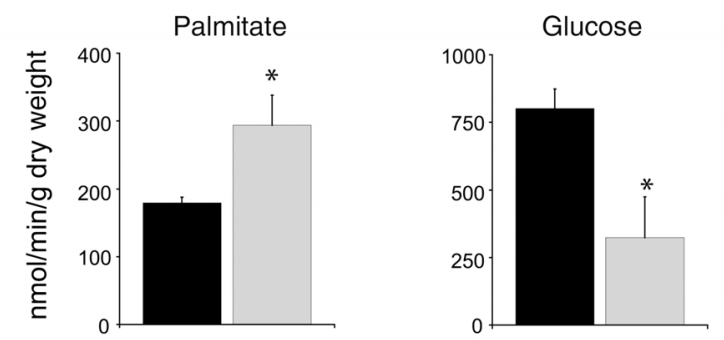

Figure 5

Myocardial palmitate utilization is increased and FDG reduced in MHC-PPAR mice. (a) The top two panels contain representative images of ${ }^{11} \mathrm{C}$-palmitate and ${ }^{18} \mathrm{~F}$-FDG uptake into myocardium as assessed by microPET in NTG and MHC-PPAR mice following a 6-hour fast. The relative amount of tracer uptake into the mouse heart 15 seconds after bolus injection of ${ }^{11} \mathrm{C}$-palmitate or ${ }^{18} \mathrm{~F}$-FDG into the jugular vein is indicated by the color scale (0-100). The arrows indicate the cardiac field. As shown in the upper panel, the color field is increased into the red scale in the hearts of MHC-PPAR mice infused with ${ }^{11} \mathrm{C}$-palmitate, which is indicative of enhanced myocardial uptake of fatty acid. Conversely, uptake of ${ }^{18} \mathrm{~F}$-FDG is substantially lower in hearts of MHC-PPAR mice than in those of NTG littermates. The graphs below the images contain time-activity curves for the cardiac fields. (b) Myocardial palmitate oxidation is increased and glucose oxidation reduced in MHC-PPAR mice. The oxidation of $\left[9,10-{ }^{3} \mathrm{H}\right]$ palmitate and $\left[\mathrm{U}-{ }^{14} \mathrm{C}\right]$ glucose was assessed in isolated working hearts (as described in Methods) of MHC-PPAR (line 402-2; $n=6$ ) or NTG littermate $(n=4)$ mice fed control chow. Bars represent mean ( \pm SEM) oxidation rates expressed as nanomoles of substrate oxidized per minute per gram dry mass. ${ }^{*} P<0.05$ versus NTG littermate mice. 

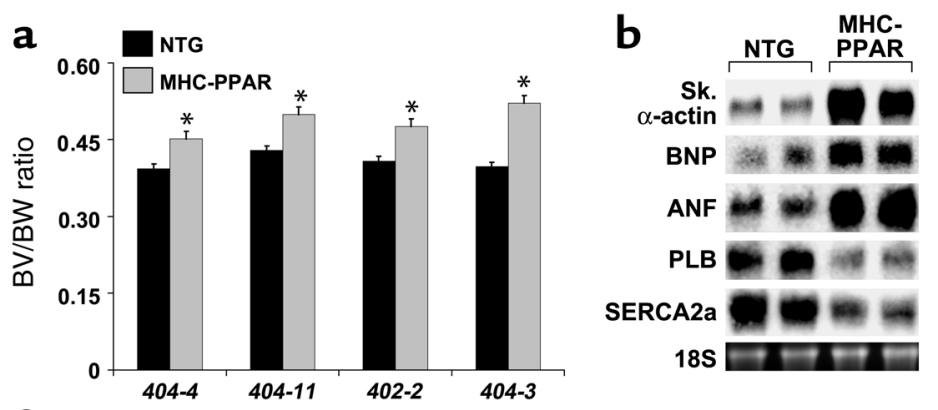

\section{Figure 6}

Induction of the cardiac hypertrophic growth gene regulatory program and left ventricular dysfunction in MHC-PPAR mice. (a) Bars represent mean biventricular-to-body-weight (BV/BW) ratios ( $n \geq 20$ for each group) for male and female MHC-PPAR and NTG littermate mice. All mice were between 2 and 4 months of age. ${ }^{*} P<0.05$ versus NTG littermate mice. (b) Representative autoradiographs of Northern blot analyses performed with total RNA isolated from cardiac ventricle from 6-week-old NTG or MHC-PPAR (404-3 line) mice using cDNA probes for skeletal (Sk.) $\alpha$-actin, brain-type natriuretic peptide (BNP), atrial natriuretic factor (ANF), phospholamban (PLB), and sarcoplasmic/endoplasmic reticulum $\mathrm{Ca}^{2+}$ ATPase $2 \mathrm{a}$ (SERCA2a). (c) Ventricular dysfunction in MHC-PPAR mice is related to transgene expression level. Representative two-dimensional guided Mmode echocardiographic images of the left ventricle obtained from the parasternal view at the midventricular level of NTG and four different transgenic lines of female mice at 2 months of age.

The cardiac function of MHC-PPAR mice was evaluated to determine whether, as speculated, energy metabolic derangements lead to cardiomyopathy. We found that the MHC-PPAR hearts exhibit ventricular hypertrophy in association with activated expression of hypertrophic gene markers. Moreover, as occurs in severe forms of diabetic cardiomyopathy, the MHCPPAR mouse lines with the highest level of transgene expression developed LV chamber dilatation and systolic ventricular dysfunction. Taken together, these results strongly suggest that the energy metabolic shifts of the diabetic heart become maladaptive and contribute to the development of cardiac dysfunction.

An important question raised by our results relates to the mechanistic link between altered myocardial energy metabolism and cardiac dysfunction in the diabetic heart. It is possible that in the context of high-level fatty acid uptake and mitochondrial $\beta$-oxidation, toxic lipid intermediates accumulate within cardiac myocytes. For example, a mismatch between the rates of fatty acid import and oxidation may result in cardiac myocyte lipid accumulation such as that observed in MHC-PPAR mice, which could cause contractile dysfunction or cell death. Indeed, previous studies have shown that lipid often accumulates in the myocardium of diabetic animals (7-9). A recent study by Chiu et al. (10) has shown that myocardial lipid accretion due to increased fatty acid uptake leads to myocyte apoptosis and cardiomyopathy - a "lipotoxic" effect. However, we did not find evidence for increased myocardial apoptosis in the MHC-PPAR mice (data not shown). Altered mitochondrial function could also play a role in the cardiac dysfunction observed in the MHC-PPAR mice. For example, increased mitochondrial flux may lead to the generation of harmful reactive oxygen species. The observed increase in UCP3 gene expression in the MHC-PPAR and diabetic heart (25) may also relate to the development of cardiac dysfunction. However, the role of UCP3 as a bona fide mitochondrial respiratory uncoupler in heart remains unclear. Increased respiratory uncoupling could actually be adaptive in the setting of increased mitochondrial flux, such as occurs in the diabetic and MHC-PPAR heart. Lastly, reliance on FAO for ATP production, which results in higher mitochondrial oxygen consumption costs compared with glycolysis and glucose oxidation, could also contribute to ventricular dysfunction.

Reduced myocardial glucose utilization may also account for the observed cardiac dysfunction in the MHC-PPAR mice. Previous studies of the ischemic and reperfused heart indicate that a reduction in glycolysis and glucose oxidation is associated with diminished ventricular function (30-32). In addition, the hearts of GLUT4-deficient mice develop profound dysfunction during ischemia (33). The mechanisms for these observations are unknown but could involve

Table 2

LV function in MHC-PPAR mice

\begin{tabular}{|c|c|c|c|c|c|c|c|c|}
\hline & \multicolumn{2}{|c|}{$404-3$ line } & \multicolumn{2}{|c|}{$402-2$ line } & \multicolumn{2}{|c|}{ 404-11 line } & \multicolumn{2}{|c|}{$404-4$ line } \\
\hline & NTG & MHC-PPAR & NTG & MHC-PPAR & NTG & MHC-PPAR & NTG & MHC-PPAR \\
\hline $\mathrm{HR}$ & $678 \pm 12$ & $628 \pm 5$ & $631 \pm 18$ & $649 \pm 11$ & $673 \pm 27$ & $695 \pm 22$ & $646 \pm 18$ & $599 \pm 50$ \\
\hline LVPWd & $0.72 \pm 0.01$ & $0.81 \pm 0.07^{\mathrm{A}}$ & $0.70 \pm 0.02$ & $0.80 \pm 0.01^{\mathrm{A}}$ & $0.72 \pm 0.03$ & $0.74 \pm 0.02$ & $0.66 \pm 0.03$ & $0.87 \pm 0.23$ \\
\hline LVIDd & $3.3 \pm 0.04$ & $3.6 \pm 0.04 \mathrm{~A}$ & $3.2 \pm 0.07$ & $3.2 \pm 0.05$ & $3.3 \pm 0.10$ & $3.5 \pm 0.5$ & $3.6 \pm 0.3$ & $3.5 \pm 0.1$ \\
\hline LVIDs & $1.4 \pm 0.03$ & $2.1 \pm 0.05^{\mathrm{A}}$ & $1.4 \pm 0.04$ & $1.5 \pm 0.05$ & $1.4 \pm 0.07$ & $1.8 \pm 0.16$ & $1.2 \pm 0.37$ & $1.6 \pm 0.10$ \\
\hline RWT & $0.44 \pm 0.01$ & $0.45 \pm 0.01$ & $0.46 \pm 0.02$ & $0.51 \pm 0.01$ & $0.45 \pm 0.03$ & $0.43 \pm 0.02$ & $0.37 \pm 0.02$ & $0.50 \pm 0.01$ \\
\hline FS & $58.5 \pm 0.7$ & $43.2 \pm 1^{\mathrm{A}}$ & $57.2 \pm 1$ & $52.2 \pm 1^{A}$ & $57.4 \pm 1.4$ & $49.6 \pm 3^{A}$ & $58 \pm 1$ & $56 \pm 3$ \\
\hline
\end{tabular}

Values represent mean $\left( \pm\right.$ SEM) ( $n \geq 7$ for each group). ${ }^{A} P<0.05$ compared with NTG littermate control mice. HR, heart rate; LVPWd, left ventricle posterior wall thickness at diastole; LVIDd, LV internal diameter at diastole; LVIDs, LV internal diameter at systole; RWT, relative wall thickness; FS, fractional shortening. 
distinct intracellular channeling routes of ATP produced by glucose oxidation or effects related to oxygen consumption efficiency.

The MHC-PPAR hearts exhibit several interesting lipid metabolic alterations in addition to the expected increase in FAO rates. First, the microPET studies demonstrated increased myocardial palmitate uptake. Secondly, TAGs accumulate in MHC-PPAR cardiac myocytes following a short-term fast, which serves to acutely increase circulating NEFA levels. This latter observation is interesting because TAG accumulates in the diabetic heart $(7,8)$. The results of the PET and fasting studies suggest that increased myocardial fatty acid uptake leads to a positive myocyte lipid balance in the MHC-PPAR heart. However, the mechanism involved in the increased myocardial fatty acid uptake remains unknown, because the fasting-induced increase in the expression of proteins involved in fatty acid import was not significantly greater in MHC-PPAR hearts. In addition, we cannot exclude the possibility that lipid synthesis pathways, including GPAT and DGAT, are activated directly by PPAR $\alpha$ in the MHC-PPAR heart. However, we have not found evidence that GPAT or DGAT is a PPAR $\alpha$ target gene based on expression levels in fasted PPAR $\alpha^{-/-}$mice (B.N. Finck and D.P. Kelly, unpublished data).

A surprising observation in this study relates to the dramatically altered expression of genes involved in glucose transport, glycolysis, and glucose oxidation in the hearts of MHC-PPAR mice. The inhibitory effects of FAO on glucose utilization in heart were first described as the "glucose fatty-acid cycle" by Randle et al. (34). This effect involves inhibition of the pyruvate dehydrogenase complex by elevated acetyl-CoA/CoA and $\mathrm{NADH} / \mathrm{NAD}^{+}$ratios generated by increased $\beta$-oxidative flux. However, the reduction in myocardial glucose utilization observed in MHC-PPAR mice likely involves mechanisms distinct from that proposed for the Randle cycle, because it includes a gene regulatory component and involves effects on glucose transport. Fatty acid-induced insulin resistance has been well described and has been proposed to play a role in the development of type 2 diabetes $(35,36)$. Recent evidence indicates that fatty acid-induced insulin resistance involves alterations at the level of glucose transport secondary to derangements in insulin signaling $(37,38)$. Our results suggest that glucose transport can be inhibited in heart as a result of primary alterations in cellular lipid metabolism driven by PPAR $\alpha$. Consistent with the notion that increased cellular lipid uptake can serve as the initial trigger for alterations in insulin sensitivity, Kim et al. (39) recently demonstrated that transgenic overexpression of lipoprotein lipase in skeletal muscle caused insulin resistance in nondiabetic mice.

The derangements in cardiac glucose utilization we observed in MHC-PPAR mice include a gene regulatory component. It is unlikely that all of the observed gene regulatory effects occur directly via PPAR $\alpha$, because the GLUT4 and PFK genes are not known
PPAR $\alpha$ targets. However, expression of PDK4 has been shown to be activated by PPAR $\alpha$ ligands (40). It is likely that GLUT4 and PFK gene expression is repressed via PPAR $\alpha$-independent transcriptional regulatory pathways linked to alterations in cellular energy metabolism induced by PPAR $\alpha$. The observation that glucose uptake and utilization can be altered in heart secondary to PPAR $\alpha$-mediated increases in fatty acid utilization suggests the intriguing possibility that some forms of insulin resistance or type 2 diabetes could be due to alterations in components of the PPAR $\alpha$ regulatory complex or downstream genes involved in cellular fatty acid utilization.

\section{Acknowledgments}

Special thanks to Richard Boriak for technical assistance with the CPT I assays, Terry Sharp for her assistance with the microPET studies, and Mary Wingate for help with manuscript preparation. We thank J.E. Schaffer, R.V. Farese, M. Mueckler, R.A. Harris, E.G. Kranias, and T. Hashimoto for cDNAs and antibodies. The histologic studies were performed in the Digestive Diseases Research Core Center at Washington University. B.N. Finck is supported by an individual National Research Service Award (grant F32 HL67575) from the National Heart, Lung, and Blood Institute (NIH). This work was supported by NIH grants RO1 DK45416, P30 DK56341, P30 DK52574, PO1 HL5727805, PO1 HL13851, and JDFI 996003.

1. Kannel, W.B., Hjortland, M., and Castelli, W.P. 1974. Role of diabetes in congestive heart failure: the Framingham study. Am. J. Cardiol. 34:29-34.

2. Rubler, S., et al. 1972. New type of cardiomyopathy associated with glomerulosclerosis. Am. J. Cardiol. 30:595-602.

3. Stanley, W.C., Lopaschuk, G.D., and McCormack, J.G. 1997. Regulation of energy substrate metabolism in the diabetic heart. Cardiovasc. Res. 34:25-33.

4. Rodrigues, B., Cam, M.C., and McNeill, J.H. 1995. Myocardial substrate metabolism: implications for diabetic cardiomyopathy. J. Mol. Cell. Cardiol. 27:169-179.

5. Neely, J.R., Rovetto, M.J., and Oram, J.F. 1972. Myocardial utilization of carbohydrate and lipids. Prog. Cardiovasc. Dis. 15:289-329.

6. Lopaschuk, G.D., and Spafford, M. 1989. Response of isolated working hearts to fatty acids and carnitine palmitoyltransferase 1 inhibition during reduction of coronary flow in acutely and chronically diabetic rats. Circ. Res. 65:378-387.

7. Murthy, V.K., and Shipp, J.C. 1977. Accumulation of myocardial triacylglycerols in ketotic diabetes. Diabetes. 26:222-229.

8. Paulson, D.J., and Crass, M.F. 1982. Endogenous triacylglycerol metabolism in diabetic heart. Am. J. Physiol. 242:1084-1094.

9. Zhou, Y.T., et al. 2000. Lipotoxic heart disease in obese rats: implications for human disease. Proc. Natl. Acad. Sci. USA. 97:1784-1789.

10. Chiu, H.-C., et al. 2001. A novel mouse model of lipotoxic cardiomyopathy. J. Clin. Invest. 107:813-822.

11. Barger, P.M., and Kelly, D.P. 2000. PPAR signaling in the control of cardiac energy metabolism. Trends Cardiovasc. Med. 10:238-245.

12. Lee, S.S.T., et al. 1995. Targeted disruption of the $\alpha$ isoform of the peroxisome proliferator-activated receptor gene in mice results in abolishment of the pleiotropic effects of peroxisome proliferators. Mol. Cell. Biol. 15:3012-3022.

13. Djouadi, F., et al. 1998. A gender-related defect in lipid metabolism and glucose homeostasis in peroxisome proliferator-activated receptor $\alpha$ deficient mice. J. Clin. Invest. 102:1083-1091.

14. Aoyama, T., et al. 1998. Altered constitutive expression of fatty acidmetabolizing enzymes in mice lacking the peroxisome proliferator-activated receptor $\alpha(\mathrm{PPAR} \alpha)$. J. Biol. Chem. 273:5678-5684.

15. Leone, T.C., Weinheimer, C.J., and Kelly, D.P. 1999. A critical role for the peroxisome proliferator-activated receptor alpha $(\operatorname{PPAR} \alpha)$ in the cellular fasting response: the PPAR $\alpha$-null mouse as a model of fatty acid oxidation disorders. Proc. Natl. Acad. Sci. USA. 96:7473-7478.

16. Kersten, S., et al. 1999. Peroxisome proliferator-activated receptor $\alpha$ 
mediates the adaptive response to fasting. J. Clin. Invest. 103:1489-1498.

17. Asayama, K., et al. 1999. Increased peroxisomal fatty acid $\beta$-oxidation and enhanced expression of peroxisome proliferator-activator receptor$\alpha$ in diabetic rat liver. Mol. Cell. Biochem. 194:227-234.

18. Memon, R.A., et al. 2000. Up-regulation of peroxisome proliferator-activated receptors (PPAR-alpha) and PPAR-gamma mRNA expression in the liver in murine obesity: troglitazone induces expression of PPARgamma-responsive adipose tissue-specific genes in the liver of obese diabetic mice. Endocrinology. 141:4021-4031.

19. Kroetz, D.L., Yook, P., Costet, P., Bianchi, P., and Pineau, T. 1998. Peroxisome proliferator-activated receptor $\alpha$ controls the hepatic CYP4A induction adaptive response to starvation and diabetes. J. Biol. Chem. 273:31581-31589.

20. Belke, D.D., Larsen, T.S., Lopaschuk, G.D., and Severson, D.L. 1999. Glucose and fatty acid metabolism in the isolated working mouse heart. Am. J. Physiol. 277:R1210-R1217.

21. Cresci, S., Wright, L.D., Spratt, J.A., Briggs, F.N., and Kelly, D.P. 1996. Activation of a novel metabolic gene regulatory pathway by chronic stimulation of skeletal muscle. Am. J. Physiol. 270:C1413-C1420.

22. Esser, V., Brown, N.F., Cowan, A.T., Foster, D.W., and McGarry, J.D. 1996 Expression of a cDNA isolated from rat brown adipose tissue and heart identifies the product as the muscle isoform of carnitine palmitoyltransferase I (M-CPT I). J. Biol. Chem. 271:6972-6977.

23. Han, X., and Gross, R.W. 2001. Quantitative analysis and molecular species finger printing of triglyceride molecular species directly from lipid extracts of biological samples by electrospray ionization tandem mass spectrometry. Anal. Biochem. 295:88-100.

24. Young, M.E., et al. 2001. Uncoupling protein 3 transcription is regulated by peroxisome proliferator-activated receptor $\alpha$ in the adult rodent heart. FASEB J. 15:833-845.

25. Depre, C., et al. 2000. Streptozotocin-induced changes in cardiac gene expression in the absence of severe contractile dysfunction. J. Mol. Cell. Cardiol. 32:985-996.

26. Belke, D.D., Larsen, T.S., Gibbs, E.M., and Severson, D.L. 2000. Altered metabolism causes cardiac dysfunction in perfused hearts from diabetic (db/db) mice. Am. J. Physiol. 279:E1104-E1113.

27. Wu, P., et al. 1998. Starvation and diabetes increase the amount of pyruvate dehydrogenase kinase isoenzyme 4 in rat heart. Biochem. $J$
329:197-201.

28. Ahmed, S.S., Jaferi, G.A., Narang, R.M., and Regan, T.J. 1975. Preclinical abnormality of left ventricular function in diabetes mellitus. Am. Heart J. 89:153-158

29. Dillmann, W.H. 1988. Diabetes mellitus-induced changes in the concentration of specific mRNAs and proteins. Diabetes Metab. Rev. 4:789-797.

30. Taegtmeyer, H., Goodwin, G.W., Doenst, T., and Frazier, O.H. 1997. Substrate metabolism as a determinant for postischemic functional recovery of the heart. Am. J. Cardiol. 80:3A-10A.

31. Lewandowski, E.D., and White, L.T. 1995. Pyruvate dehydrogenase influences postischemic heart function. Circulation. 91:2071-2079.

32. McVeigh, J.J., and Lopaschuk, G.D. 1990. Dichloroacetate stimulation of glucose oxidation improves recovery of ischemic rat hearts. Am. J. Physiol. 259:H1079-H1085.

33. Tian, R., and Abel, E.D. 2001. Responses of GLUT4-deficient hearts to ischemia underscore the importance of glycolysis. Circulation. 103:2961-2966.

34. Randle, P.J., Hales, C.N., and Garland, P.B. 1963. The glucose fatty-acid cycle. Its role in insulin sensitivity and the metabolic disturbances of diabetic mellitus. Lancet. 1:785-789.

35. Frayne, K.N. 1993. Insulin resistance and lipid metabolism. Curr. Opin Lipidol. 4:197-204.

36. McGarry, J.D. 1992. What if Minkowski had been ageusic? An alternative angle on diabetes. Science. 258:766-770.

37. Dresner, A., et al. 1999. Effects of free fatty acids on glucose transport and IRS-1-associated phosphatidylinositol 3-kinase activity. J. Clin. Invest. 103:253-259.

38. Griffin, M.E., et al. 1999. Free fatty acid-induced insulin resistance is associated with activity of protein kinase $\mathrm{C}$ theta and alterations in the insulin signaling cascade. Diabetes. 48:1270-1274.

39. Kim, J.K., et al. 2001. Tissue-specific overexpression of lipoprotein lipase causes tissue-specific insulin resistance. Proc. Natl. Acad. Sci. USA. 98:7522-7527.

40. Wu, P., Inskeep, K., Bowker-Kinley, M.M., Popov, K.M., and Harris, R.A. 1999. Mechanism responsible for inactivation of skeletal muscle pyruvate dehydrogenase complex in starvation and diabetes. Diabetes. 48:1593-1599. 